\title{
Medo de contrair COVID-19: Estudo de validação da Fear of Contracting COVID-19 Scale em adolescentes portugueses
}

\section{Fear of Contracting COVID-19: A validation study of the Fear of Contracting COVID-19 Scale in Portuguese adolescents}

\author{
Maria de Fátima Sousa Ramos (1) \\ Ana Galhardo $(1,2)$ \\ Marina Cunha $(1,2)$ \\ Ilda Massano-Cardoso $(1,3,4)$ \\ (1) Instituto Superior Miguel Torga, Coimbra, Portugal \\ (2) Universidade de Coimbra, CINEICC, Faculdade de Psicologia e de Ciências da Educação da Universidade de Coimbra, Portugal \\ (3) Universidade de Coimbra, Faculdade de Medicina \\ (4) Universidade de Coimbra, CEISUC, Faculdade de Economia da Universidade de Coimbra
}

Recebido: 22/06/2021; Revisto: 18/07/2021; Aceite: 21/10/2021.

https://doi.org/10.31211/rpics.2021.7.2.215

Check for updates

\begin{abstract}
Resumo
Introdução: Face às circunstâncias de pandemia, o medo pode emergir como resultado da incerteza, da possibilidade de ser contagiado pelo SARS-Cov 2, ou contagiar outras pessoas, podendo ainda estar associado a sintomas psicopatológicos e a um impacto na qualidade de vida, relacionados com a pandemia pela COVID-19. Objetivo: A presente investigação pretendeu examinar a estrutura fatorial e propriedades psicométricas da Fear of Contracting COVID-19 Scale (FCCS) em adolescentes portugueses e analisar a relação entre o medo de contrair COVID-19 e os níveis de depressão, ansiedade e stresse, bem como com a qualidade de vida neste grupo etário. Métodos: A amostra foi constituída por 269 adolescentes (137 rapazes e 132 raparigas), com idades compreendidas entre os 11 e os 16 anos, a frequentar o $3^{\circ}$ ciclo do ensino básico. Os participantes preencheram um questionário sociodemográfico, a FCCS, as Escalas de Depressão, Ansiedade e Stress (EADS-21), e o KIDSCREEN-10, enquanto medida da qualidade de vida em adolescentes. Resultados: A FCCS revelou, tal como na sua versão original para adultos, uma estrutura unidimensional, com bons indicadores de ajustamento e boa consistência interna. O medo de contrair COVID-19 mostrou-se associado com sintomas de ansiedade, depressão e stresse, mas não evidenciou uma relação com a qualidade de vida nos adolescentes. A análise de diferenças entre rapazes e raparigas mostrou que estas últimas apresentam mais medo de contrair COVID-19, mais sintomas de ansiedade, depressão e stresse e uma pior perceção da sua qualidade de vida. Conclusões: A Fear of Contracting COVID-19 Scale mostrou ser adequada para uso com adolescentes. O medo de contrair COVID-19, ainda que associado a sintomas psicopatológicos de ansiedade, depressão e stresse, parece não estar relacionado com a qualidade de vida dos adolescentes.
\end{abstract}

Palavras-Chave: Medo de contrair COVID-19; Ansiedade; Depressão; Stresse; Qualidade de Vida; Adolescentes.

\section{DI\&D | ISMT}

rpics@ismt.pt

https://rpics.ismt.pt
Maria de Fátima de S. Ramos

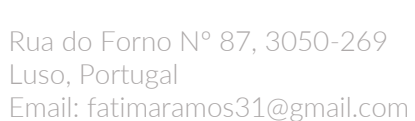




\section{Abstract}

Introduction: Given the pandemic circumstances, fear may emerge due to uncertainty, the possibility of being infected by SARS-Cov 2 or infecting others. It may also be associated with psychopathological symptoms and impact quality of life resulting from the COVID-19 pandemic. Objective: The current study aimed to examine the factor structure and psychometric properties of the Fear of Contracting COVID-19 Scale (FCCS) in Portuguese adolescents and analyze the relationship between fear of contracting COVID-19 and depression, anxiety and stress symptoms, and with the quality of life of this age group. Method: The sample comprised 269 adolescents (137 boys and 132 girls), aged between 11 and 16 years old, attending the $3 r d$ cycle of basic education. Participants completed a sociodemographic questionnaire, the FCCS, the Depression, Anxiety and Stress Scales (DASS-21), and the KIDSCREEN-10, as a measure of the quality of life in adolescents. Results: The FCCS revealed, as in its original version for adults, a one-dimensional structure, with good adjustment indicators and good reliability. The fear of contracting COVID-19 was associated with anxiety, depression, and stress symptoms, but it did not show a relationship with the adolescents' quality of life. Gender differences showed that the girls present higher levels of fear of contracting COVID-19, more anxiety, depression and stress symptoms, and a worse perception of their quality of life. Conclusions: The Fear of Contracting COVID-19 Scale showed to be suitable for use with adolescents. The fear of contracting COVID-19, although associated with psychopathological symptoms of anxiety, depression and stress, does not seem to be related to the quality of life of adolescents.

Keywords: Fear of contracting COVID-19; Anxiety; Depression; Stress; Quality of Life; Adolescents.

\section{Introdução}

Em dezembro de 2019, uma nova estirpe de um coronavírus foi detetada em Wuhan, província de Hubei, China. Esse vírus foi identificado pela World Health Organization (WHO, 2020) como SARS-CoV-2 e a doença por ele provocada como COVID-19 (Coronavírus Disease-19). A COVID-19 transmitiu-se rapidamente por todo o mundo, levando esta organização a decretar, em março de 2020, um estado de pandemia (WHO, 2020).

A pandemia causada pelo novo coronavírus traduz a maior emergência de saúde pública que a comunidade internacional enfrenta em décadas (Schmidt et al., 2020). Além das preocupações relacionadas com a saúde física, também acarreta preocupações quanto ao sofrimento psicológico que pode ser experienciado pela população (Schmidt et al., 2020). A rápida disseminação do novo coronavírus por todo o mundo, as incertezas sobre como controlar a doença e sobre a gravidade da COVID-19, além da imprevisibilidade acerca do tempo de duração da pandemia e o surgimento de diferentes estirpes, constituem-se como fatores de risco para a saúde mental da população geral (Schmidt et al., 2020).

Durante as epidemias, o número de pessoas cuja saúde mental é afetada tende a ser maior que o número de pessoas afetadas pela infeção causada pela doença em si (Ornell et al., 2020). Em situações de crise, tende a existir uma multiplicidade de preocupações que emergem, principalmente quando as crianças ou jovens se veem forçadas a conviver repentinamente com uma realidade do dia-a-dia diferente daquela que conhecem (Alcobia et al., 2020). Em Portugal, como em quase todo o mundo, as medidas de contenção adotadas como forma de prevenir a propagação da doença ficaram marcadas socialmente pela quarentena ou confinamento obrigatório, implicando a mudança nas rotinas de vida diária (e.g., teletrabalho, aulas online), a separação das pessoas das suas famílias e da comunidade em geral. Como consequência destas medidas e, com base no conhecimento sobre o impacto das situações epidémicas anteriores, antecipa-se que a experiência de isolamento, a incerteza e as preocupações em relação ao 
que o futuro reserva terão um impacto psicológico significativo, a curto e longo prazo, na saúde mental das populações que vivenciam a pandemia (e.g., Cao et al., 2020; Wang et al., 2020). Alguns fatores, como a duração da quarentena, associada ao medo da infeção ou medo da transmissão a outros, o acesso a informações inadequadas (fake news), a diminuição dos contactos sociais, a perda financeira e o estigma em relação às pessoas contaminadas ou familiares, têm sido referidos como preditores de problemas de saúde mental, estando associados a um aumento significativo do sofrimento psicológico (Brooks et al., 2020). Este impacto psicológico tem sido caracterizado na literatura por um aumento de sintomas de stresse pós-traumático, ansiedade, irritabilidade, perturbações do sono e sintomas depressivos (Ribeiro et al., 2020).

Com efeito, a COVID-19 foi percecionada em todo o mundo como uma grande ameaça para a saúde e um perigo para a economia global, afetando significativamente a vida das pessoas, influenciando o seu comportamento diário e causando sentimentos de pânico, ansiedade, depressão e medo intensos (Jiao et al., 2020). Embora as crianças e adolescentes pareçam ser menos vulneráveis do que os adultos em relação ao desenvolvimento de formas graves da COVID-19, relatórios iniciais de áreas chinesas atingidas pela doença indicaram que crianças e adolescentes sofreram um grande impacto psicológico, manifestando problemas comportamentais, bem como respostas de medo e incerteza (Jiao et al., 2020). De facto, o impacto psicológico negativo desta situação de pandemia, nas crianças e adolescentes, relaciona-se sobretudo com problemas emocionais e comportamentais, mais concretamente, défice de atenção, irritabilidade, agitação, medo de fazer perguntas sobre a situação pandémica, ansiedade de separação dos familiares, perturbações do sono, anorexia e queixas somáticas (Jiao et al., 2020). De referir que o stresse psicológico relacionado com o impacto da pandemia acarreta efeitos negativos na saúde das crianças e adolescentes, verificando-se a manifestação de sintomas de ansiedade, depressão, letargia, dificuldades na interação social e apetite reduzido (Singh et al., 2020). Segundo Wang et al. (2020), o tempo prolongado do período de confinamento, o medo de contrair a COVID-19, a desinformação relativa à doença nas redes sociais, a ausência de contacto pessoal com familiares, pares, amigos e professores, a limitação de espaço pessoal nas suas casas e as dificuldades económicas foram as principais causas apontadas para a existência de stresse. Embora as crianças e jovens apresentem formas menos graves da COVID-19, não deixam de ser suscetíveis às consequências negativas na saúde mental relacionadas com a pandemia. Períodos de prolongada exposição ao stresse, privação da estimulação social e dos cuidados de saúde, podem significar implicações significativas na saúde mental das crianças e jovens, tendo repercussões no seu bem-estar (Hamoda et al., 2021).

Para compreender as repercussões psicológicas e psiquiátricas de uma pandemia, as emoções envolvidas devem ser consideradas e observadas, como é o caso do medo. O medo é um mecanismo adaptativo, fundamental para a sobrevivência, e que envolve vários processos biológicos de preparação para uma resposta a eventos potencialmente ameaçadores (Ornell et al., 2020). Trata-se de uma emoção básica, presente desde o nascimento, muito comum na infância e na adolescência (Schoen \& Vitalle, 2012), e que se constitui como uma reação emocional que surge da interpretação que o indivíduo faz perante uma situação como perigosa e ameaçadora do seu bem-estar (Reeve, 2014). Magalhães (2007) identifica na resposta de medo algumas características como a ansiedade, apreensão, nervosismo, pavor e preocupação, referindo que esta emoção pode ser extremamente breve, mas que pode, também, durar um longo período. Segundo este autor, a intensidade do medo depende da ameaça, ou seja, da avaliação que o indivíduo faz relativamente ao facto de esta ameaça ser imediata, ou estar pendente, e dos 
recursos que perceciona ter disponíveis para lidar com essa ameaça ou perigo. De acordo Rogers (1975), a função principal da emoção do medo é a de proteger a integridade física e psicológica do indivíduo, motivando-o para se libertar ou fugir de situações potencialmente temíveis. Na maioria das vezes, o medo é uma reação adaptativa na qual existe um fluxo de energia que pode ser usada em qualquer ação necessária para enfrentar o perigo, englobando respostas motoras e neurovegetativas (e.g., taquicardia, sudação, tensão muscular) (Schoen \& Vitalle, 2012).

Em tempo de pandemia, o medo aumenta os níveis de ansiedade e stresse dos indivíduos saudáveis e intensifica os sintomas daqueles que já manifestam perturbações psiquiátricas (Ornell et al., 2020). O medo de ser infetado por um vírus potencialmente fatal, de rápida disseminação, cujas origens, natureza e curso ainda são pouco conhecidos, acaba por afetar o bem-estar psicológico de muitas pessoas (Salvaterra \& Chora, 2021). No entanto, há que ter em conta que o medo ou a perceção de risco em relação à infeção se constitui como um preditor para a maior probabilidade de adotar comportamentos protetores de lavagem das mãos ou de distanciamento social (Wise et al., 2020).

Sintomas de depressão, ansiedade e stresse face à pandemia têm sido identificados na população geral, sendo estes mais prevalentes no sexo feminino (Nabuco, 2020; Paulino, 2021; Wang et al., 2020).

A ansiedade, embora seja uma característica adaptativa do ser humano que nos permite manter um estado de alerta, o que pode ser positivo na proteção em relação à contaminação pelo novo coronavírus (pelos comportamentos de proteção adotados), pode, também, sendo excessiva, originar medos irracionais, tristeza e confusão, gerando prejuízo ao indivíduo e repercutindo-se na sua família, em especial, nas crianças e jovens (Salvaterra \& Chora, 2021). A ansiedade, quando atinge uma intensidade extrema, pode provocar mal-estar intenso e alteração no funcionamento social, profissional, académico e ocupacional dos indivíduos, sendo estas manifestações sinal de alarme (Correia \& Brites, 2020). Nas crianças e adolescentes é particularmente importante ter em conta o impacto que a ansiedade tem na aprendizagem, na frequência escolar, nas relações com familiares e nas relações com os pares (Brito, 2011).

As medidas de contenção da pandemia podem, também, constituir fatores de risco para a saúde mental, dado o sofrimento psicológico experienciado pelas famílias, devido às medidas de confinamento e às mudanças nas rotinas e nas relações familiares (Salvaterra \& Chora, 2021). Segundo Merino e Agustin (2020), no período de confinamento os níveis de stresse e ansiedade tendem a aumentar de acordo com a idade e as responsabilidades associadas, havendo uma relação entre stresse e ansiedade e entre essas variáveis e o número de dias de confinamento.

As restrições governamentais colocadas em vigor para controlar a propagação do vírus levaram a um amplo isolamento social, que teve consequências profundas para a saúde mental (Magson et al., 2020). De acordo com Magson et al. (2020) essas restrições têm sido desafiadoras para as pessoas de todas idades, mas particularmente difíceis para os adolescentes, que nesta etapa desenvolvimental dependem fortemente das suas ligações com os pares para a obtenção de suporte emocional e para o seu desenvolvimento social (Magson et al., 2020; Miliauskas \& Faus, 2020).

De acordo com a World Health Organization, a qualidade de vida corresponde à perceção que um indivíduo tem sobre a sua posição na vida, dentro do contexto dos sistemas de cultura e valores nos quais está inserido e em relação aos seus objetivos, expectativas, padrões e preocupações. Assim, qualidade de vida corresponde à influência da saúde física e psicológica, nível de independência, relações sociais, crenças pessoais e das suas relações com características inerentes ao respetivo meio na avaliação subjetiva da qualidade de vida individual (WHO, 1998). 
Wallander et al. (2001) considera que a qualidade de vida das crianças e adolescentes pode ser definida como a combinação do bem-estar subjetivo e objetivo, em múltiplos domínios da vida considerados salientes na cultura do próprio e no seu tempo histórico, dentro do âmbito dos padrões universais dos direitos humanos. Segundo Gaspar et al. (2006), na convenção das Nações Unidas sobre os direitos das crianças foi reconhecido o direito destas à saúde, lazer, educação e direito a um nível de vida adequado ao seu desenvolvimento físico, mental, espiritual, moral e social. Deste modo, a qualidade de vida e bem-estar das crianças implicam mais do que ausência de maus-tratos e deficits, implicam, também, força e qualidades positivas no contexto mais geral e na família da criança (Gaspar et al., 2006). Segundo Gaspar et al. (2008), a qualidade de vida em crianças e adolescentes está intimamente relacionada com a sua saúde mental e bemestar subjetivo, envolvendo a interação de múltiplas variáveis contextuais como a casa, a família, os pais, a escola, os pares, a comunidade e a sociedade em geral (Gaspar et al., 2008). A ideia de qualidade de vida não se restringe à satisfação de necessidades materiais, mas está também relacionada com valores não-materiais como inserção social, felicidade, liberdade e bem-estar, entre outros. Esta é vista como um construto multidimensional que inclui as dimensões física, que compreende a perceção do indivíduo sobre a sua condição física; psicológica, ou seja, a perceção do indivíduo sobre a sua condição afetiva e cognitiva; e social, que traduz a perceção do indivíduo sobre os relacionamentos sociais (Soares et al., 2011).

Na sequência do reconhecimento do impacto da pandemia pela COVID-19 em indicadores de saúde mental, emergiu a necessidade de medidas válidas e fidedignas que possibilitassem uma melhor compreensão desse mesmo impacto. Assim, com o intuito de avaliar o medo de infeção pela COVID-19, foram desenvolvidos alguns instrumentos de autorresposta, como é o caso da Fear of COVID-19 Scale (FCV-19S; Ahorsu et al., 2020). A FCV-19S é uma escala composta por sete itens, que tem vindo a ser traduzida e estudada em diversas línguas, evidenciando boas propriedades psicométricas. Mais recentemente, esta escala conta com uma versão em português (Soares et al., 2021). Em adolescentes, a FCV-19S foi estudada no Japão (Masuyama et al., 2020), tendo revelado uma estrutura bifatorial, integrando um fator emocional e um fator fisiológico.

Um outro instrumento desenvolvido foi a Coronavirus Anxiety Scale (Lee, 2020a), correspondendo a um instrumento breve ( 5 itens) de screening para identificação de indivíduos que apresentem níveis disfuncionais de ansiedade em relação à COVID-19. Esta escala demonstrou ser um instrumento válido e fidedigno, possibilitando a discriminação entre sujeitos com e sem níveis disfuncionais de ansiedade (Lee, 2020a).

Também a Obsession with COVID-19 Scale (Lee, 2020b) foi criada com o intuito de avaliar sinais clínicos de processos cognitivos disfuncionais relacionados com a COVID-19. Os seus quatro itens revelaram uma boa consistência interna e a análise fatorial confirmatória excelentes índices de ajustamento, sendo uma escala capacidades discriminativas do ponto de vista clínico (Lee, 2020b).

As COVID-19 Stress Scales (Taylor et al., 2020), compostas por 36 itens, foram um outro instrumento de autorresposta construído com o intuito de avaliar e melhor compreender o distress relacionado com a COVID-19. O estudo da sua estrutura fatorial demonstrou a existência de cinco fatores de stresse e ansiedade relativos à COVID-19: 1) perigo e medos de contaminação; 2) medos acerca das consequências económicas; 3) xenofobia; 4) comportamentos de verificação e procura de tranquilização; e 5) sintomas de stresse traumático relacionados com a COVID-19. Estas escalas revelaram bons indicadores de fidedignidade e de validade (Taylor et al., 2020).

Em Portugal, Trindade e Ferreira (2020) desenvolveram a Fear of Contracting COVID-19 Scale (FCCS). Esta escala destina-se a avaliar o nível de medo/preocupação experienciado nas situações identificadas 
nos seus nove itens, tendo o estudo da sua estrutura fatorial e características psicométricas sido conduzido numa amostra de pessoas com doença inflamatória intestinal. A FCCS evidenciou uma estrutura unidimensional e uma boa fidedignidade (Trindade \& Ferreira, 2020).

Na globalidade, a pandemia pela COVID-19 acarretou mudanças significativas na vida dos adolescentes, as quais poderão ter impacto na sua saúde mental e qualidade de vida. Como tal, a presente investigação pretendeu examinar a estrutura fatorial e características psicométricas da FCCS (Trindade \& Ferreira, 2020) numa amostra de adolescentes portugueses e explorar a relação entre o medo de contrair a doença COVID19, sintomas psicopatológicos (depressão, ansiedade e stresse) e os níveis de qualidade de vida em adolescentes. De notar que a escolha desta escala se prendeu com o facto de esta ter sido desenvolvida em língua portuguesa e de os itens serem generalistas, ou seja, não englobarem qualquer alusão à doença inflamatória intestinal.

\section{Método}

\section{Participantes}

A amostra, não probabilística, recolhida por conveniência, foi constituída por 269 adolescentes, 137 do sexo masculino (50,9\%) e 132 do sexo feminino (49,1\%), com idades compreendidas entre os 11 e os 16 anos $(M=13,09 ; D P=0,90)$. Relativamente aos anos de escolaridade, os participantes distribuíram-se entre $\circ 7^{\circ}$ e $8^{\circ}$ ano de escolaridade, a frequentar escolas públicas do distrito de Aveiro (município de Ílhavo). As características gerais da amostra (sexo, idade, ano de escolaridade) encontram-se apresentadas na Tabela 1.

\section{Tabela 1}

Caracterização Sociodemográfica da Amostra

\begin{tabular}{|c|c|c|c|c|c|c|}
\hline & $n$ & $M$ & $D P$ & $\%$ & Mínimo & Máximo \\
\hline Idade & & 13,09 & 0,90 & & 11 & 16 \\
\hline \multicolumn{7}{|l|}{ Escolaridade } \\
\hline $7^{\circ}$ & 158 & & & 58,70 & & \\
\hline $8^{\circ}$ & 111 & & & 41,30 & & \\
\hline \multicolumn{7}{|l|}{ Sexo } \\
\hline Masculino & Masculino & & & 50,9 & & \\
\hline Feminino & Feminino & & & 49,1 & & \\
\hline
\end{tabular}

Nota. $N=269$.

\section{Instrumentos}

$\mathrm{Na}$ presente investigação, para além de um questionário sociodemográfico, que integrou questões relativas à idade, sexo, e ano de escolaridade, foram usados os instrumentos de autorresposta que se seguem. 


\section{KIDSCREEN-10}

O KIDSCREEN-10 (Ravens-Sieberer et al., 2005; versão portuguesa de Gaspar \& Matos, 2008) é um instrumento estandardizado que avalia a qualidade de vida em crianças e adolescentes através de dez itens (e.g., "Sentiste-te triste?"; "Divertiste-te com os teus(tuas) amigos(as)?". Cada um dos dez itens é respondido numa escala de nada (1) a totalmente (5), sendo aplicável a crianças e adolescentes entre os dez e os 16 anos de idade. Relativamente à pontuação, um valor baixo neste instrumento reflete sentimentos de infelicidade, insatisfação e desadequação face aos diversos contextos da vida da criança ou adolescente, nomeadamente, família, grupo de pares e escola. Uma cotação elevada revela uma sensação de felicidade, perceção de adequação e satisfação com os seus contextos (Matos et al., 2012). A versão portuguesa do KIDSCREEN-10 mostrou uma boa consistência interna (a de Cronbach =0,78). $\mathrm{Na}$ presente amostra, o KIDSCREEN-10 mostrou um valor de alfa de Cronbach de 0,77.

\section{Escala de Ansiedade, Depressão e Stress-21 itens (EADS-21)}

A EADS-21 visam a avaliação de sintomas emocionais negativos de ansiedade (e.g., "Senti-me assustado sem ter tido uma boa razão para isso"), depressão (e.g., "Senti-me desanimado e melancólico") e stresse (e.g., "Senti que por vezes estava sensível"). A escala de ansiedade centra-se na excitação do sistema nervoso autónomo, ansiedade situacional e experiências ansiógenas subjetivas. A escala de depressão engloba sintomas de disforia, desânimo, desvalorização, anedonia e inércia. Por último, a escala de stresse foca-se na dificuldade em relaxar, na impaciência e na irritabilidade. As opções de resposta distribuem-se numa escala que varia entre não se aplicou nada a mim (0) e aplicou-se a mim a maior parte das vezes (3), representando a severidade e frequência dos sintomas experienciados nos últimos sete dias. Estados emocionais mais negativos estão associados a pontuações mais elevadas. Na versão portuguesa da EADS21 os valores do alfa de Cronbach foram respetivamente de 0,85 para a escala de depressão, de 0,74 para a de ansiedade e de 0,81 para a de stresse (Pais-Ribeiro et al., 2004). No presente estudo verificaram-se valores de alfa de Cronbach de 0,87 para escala ansiedade, 0,87 para escala depressão e 0,90 para escala de stresse.

\section{Procedimentos Metodológicos}

Num primeiro momento, procedeu-se à recolha das autorizações dos autores das versões portuguesas dos instrumentos de autorresposta para a respetiva utilização. De seguida, foi submetido o pedido de realização do estudo à Direção-Geral da Educação (DGE), tendo este sido avaliado e aprovado (pedido de realização do inquérito $n^{\circ}$ 0769500001). Na sequência desta aprovação, foi solicitada a autorização aos diretores dos estabelecimentos de ensino para efeitos de recolha de dados. Posteriormente, foram contactados os encarregados de educação dos alunos, bem como os alunos, sendo facultadas informações acerca do âmbito e objetivos da investigação, participação voluntária, anonimato e confidencialidade, sendo recolhido o consentimento informado, de acordo com os princípios éticos aplicáveis a estudos desta natureza constantes do Código Deontológico da Ordem dos Psicólogos Portugueses (2014).

A recolha de dados decorreu entre os meses de abril e maio de 2021, contando com a colaboração dos docentes responsáveis, e tendo em conta a calendarização e as orientações das escolas dos Agrupamentos de Escolas do Eixo e de Ílhavo (distrito de Aveiro). De acordo com as recomendações de Tabachnick e 
Fidell (2013) foi recolhida uma amostra com um número de participantes superior a dez indivíduos por item da FCCS.

O protocolo de instrumentos de autorresposta foi administrado em grupo, no contexto de sala de aula, seguindo todas as normas da Direção-Geral da Saúde (DGS) relativamente ao distanciamento, higiene pessoal (lavagem das mãos, desinfeção das mesmas com álcool gel e etiqueta respiratória, utilização de equipamentos de proteção individual) e higiene ambiental (limpeza, desinfeção e ventilação adequada da sala). Previamente a cada administração, os alunos foram novamente informados acerca do âmbito e objetivos do estudo, do carácter voluntário da sua participação e da obrigatoriedade de facultarem o seu consentimento informado. O preenchimento teve duração média de 15 minutos e contou com a presença do investigador para esclarecer quaisquer dúvidas e garantir respostas explícitas e independentes.

\section{Procedimentos Analíticos}

Para efeitos de análise dos dados, utilizou-se o programa Statistical Package for the Social Sciences (IBM SPSS Statistics, versão 27.0). Para a análise fatorial confirmatória (AFC) utilizou-se o software IBM SPSS AMOS. Na caracterização da amostra foram calculadas as médias e desvios padrão das variáveis contínuas e as frequências e percentagem das variáveis categoriais.

As análises conduzidas com vista a averiguar a estrutura da FCCS em adolescentes replicaram as levadas a cabo no estudo da versão original deste instrumento para adultos (Trindade \& Ferreira, 2020), nomeadamente uma análise de componentes principais $(A C P)$ e uma $A F C$. Previamente à realização da $A C P$ foram calculados o teste de Kaiser-Meyer-Olkin (KMO) e o teste de esfericidade de Bartlett. Foram considerados os valores de referência propostos por Costello e Osborne (2005) para as ciências sociais, com os pesos fatoriais a variarem entre 0,30 e 0,50 e as comunalidades a variarem entre 0,40 e 0,70.

Relativamente à qualidade do ajustamento do modelo, foram considerados os seguintes indicadores: quiquadrado normalizado ( $\chi 2 / g l$; valores < 5 são indicadores de que o modelo apresenta um bom ajustamento aos dados empíricos), o Comparative Fit Index (CFI), o Goodness of Fit Index (GFI) (valores > 0,90 são indicadores de um bom ajustamento) (Marôco, 2010), e o Standardized Root Mean Square Residual (SRMR) (valores < 0,08 são reveladores de um bom ajustamento) (Hu \& Bentler, 1999; Kline, 2005). O ajustamento local do modelo foi analisado através dos pesos de regressão estandardizados e das correlações múltiplas ao quadrado ( $\geq 0,50$ e $\geq 0,25$, respetivamente, indicam um bom ajustamento local; Marôco, 2010). A consistência interna foi calculada através do alfa de Cronbach, considerando-se valores $>0,70$ como correspondendo a uma boa consistência interna (Kline, 2000). Procedeu-se à análise da associação entre as variáveis em estudo através do coeficiente de correlação de Pearson. Segundo a classificação de Marôco (2014), um coeficiente de correlação de Pearson de valor inferior a 0,25 é fraco, entre 0,25 e 0,50 é moderado, entre 0,50 e 0,75 é forte e superior a 0,75 é muito forte. Com o intuito de examinar as diferenças entre sexos para as variáveis em estudo foi realizado o teste $t$-Student para amostras independentes. Para medir a dimensão do efeito (i.e., da magnitude das diferenças encontradas entre os grupos em comparação) calculou-se o $d$ de Cohen, sendo este interpretado de acordo com as diretrizes de Cohen (1992), ou seja, > 1 muito elevado, entre 0,5 e 1,0 elevado, entre 0,2 e 0,5 médio e, por último, < 0,2 pequeno. 


\section{Resultados}

\section{Análise Fatorial da FCCS}

Com o intuito de analisar a estrutura fatorial da FCCS na presente amostra, foram conduzidas as análises reportadas pelos autores da versão deste instrumento para adultos. Preliminarmente, foi calculado o teste de KMO $(0,86)$ e o teste de esfericidade de Bartlett $\left(\chi_{(36)}^{2}=1054,93 ; p<0,001\right)$, tendo estes revelado adequação para a condução da referida análise. A ACP revelou a existência de dois fatores com eigenvalues superiores a 1 , a explicarem $64,3 \%$ da variância total. Tal como encontrado na versão original de adultos, todos os itens saturaram no primeiro fator, pelo que também na versão para adolescentes se repetiu a análise, forçada a um fator, o qual explicou 48,8\% da variância total. Na Tabela 2 são apresentadas as médias, desvios padrões, correlações item-total e valor de alfa se item removido, bem como os pesos fatoriais e comunalidades de cada um dos itens.

O cálculo do alfa de Cronbach revelou um valor de 0,86. Como se pode observar na Tabela 2, todos os itens mostraram uma correlação com o total igual ou superior a 0,46 e a remoção de qualquer dos itens não conduziria a um aumento da consistência interna.

Uma vez mais seguindo os procedimentos levados a cabo no estudo da versão original da FCCS para a adultos, foi realizada uma análise fatorial confirmatória. Mediante a definição de correlações entre os resíduos dos itens $2 \leftrightarrow 3$, o modelo unidimensional revelou os seguintes valores de ajustamento: $C M I N / g I=4,21 ; C F I=0,93 ; G F I=0,92$ e $S R M R=0,06$. A comparação do modelo inicial com o modelo em que foram definidas as correlações entre os resíduos dos itens indicou uma diminuição do MECVI de 0,98 para 0,54.

No que se refere aos índices de ajustamento local (Tabela 2), os pesos de regressão estandardizados variaram entre 0,45 (Item 3) e 0,81(Item 5) e as correlações múltiplas ao quadrado entre 0,20 (Item 2) e 0,66 (Item 5).

\section{Relação entre o Medo de Contrair COVID-19, Sintomas Psicopatológicos e Qualidade de Vida, e Diferenças entre os Sexos}

As associações entre as variáveis em estudo são apresentadas na Tabela 3. O medo de contrair COVID19 (FCCS) correlacionou-se de forma estatisticamente significativa com os sintomas psicopatológicos de ansiedade, depressão e stresse. Já no que respeita à associação entre o medo de contrair COVID-19 e a qualidade de vida dos adolescentes não se observou uma correlação estatisticamente significativa ( $r$ = $0,05 ; p=0,414)$. 


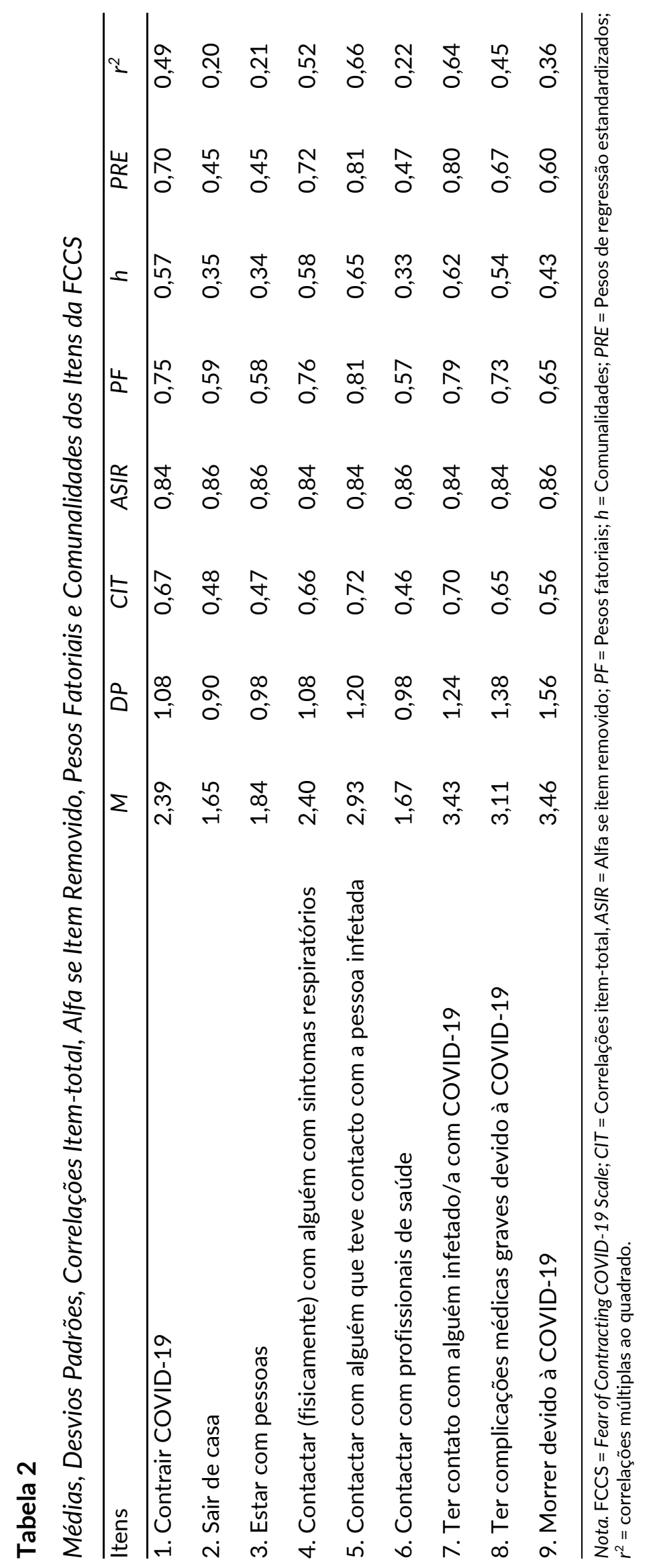




\section{Tabela 3}

Associações entre o Medo de Contrair COVID-19, Sintomas Psicopatológicos e Qualidade de Vida

\begin{tabular}{llllll}
\hline Medidas & 1 & 2 & 3 & 4 & 5 \\
\hline 1. FCCS & - & $0,26^{* *}$ & $0,17^{* *}$ & $0,25^{* *}$ & $-0,07$ \\
2. EADS-21 Ansiedade & & - & $0,78^{* *}$ & $0,82^{* *}$ & $-0,65^{* *}$ \\
3. EADS-21 Depressão & & & - & $0,79^{* *}$ & $-0,73^{* *}$ \\
4. EADS-21 Stresse & & & - & $-0,62^{* *}$ \\
5. KIDSCREEN-10 & & & & - \\
\hline
\end{tabular}

Nota. $N=269$. FCCS = Scale Fear Contracting COVID-19; EADS-21 = Escalas de Ansiedade, Depressão e Stresse.

Ao explorar se a idade se correlacionava com o medo de contrair COVID-19, verificou-se não existir uma correlação estatisticamente significativa $(r=-0,09 ; p=0,126)$.

A análise de diferenças entre rapazes e raparigas nas variáveis em estudo (Tabela 4) revelou existirem diferenças estatisticamente significativas, com as raparigas a apresentarem níveis mais elevados de medo de contrair COVID-19, assim como de sintomatologia ansiosa, depressiva e de stresse. Quanto à perceção da qualidade de vida foram encontradas diferenças estatisticamente significativas entre os sexos, verificando-se que os rapazes percecionam a qualidade de vida de maneira mais positiva que as raparigas.

\section{Tabela 4}

Comparação de Médias das Variáveis em Estudo em Função do Sexo

\begin{tabular}{|c|c|c|c|c|c|c|c|}
\hline \multirow[b]{2}{*}{ Medidas } & \multicolumn{2}{|c|}{ Masculino $n=137$} & \multicolumn{2}{|c|}{ Feminino $n=132$} & \multirow{2}{*}{$t$} & \multirow{2}{*}{$p$} & \multirow{2}{*}{$d$} \\
\hline & M & $D P$ & M & $D P$ & & & \\
\hline FCCS & 21,74 & 7,22 & 24,07 & 7,19 & $-2,65$ & 0,008 & $-0,32$ \\
\hline EADS-21 - Ansiedade & 3,26 & 4,36 & 7,02 & 5,38 & $-6,28$ & $<0,001$ & $-0,77$ \\
\hline EADS-21 - Depressão & 5,12 & 5,46 & 8,48 & 5,84 & $-4,87$ & $<0,001$ & $-0,71$ \\
\hline EADS-21 - Stresse & 4,69 & 4,44 & 8,11 & 5,19 & $-5,79$ & $<0,001$ & $-0,60$ \\
\hline KIDSCREEN - 10 & 34,77 & 6,78 & 31,04 & 6,78 & 4,51 & $<0,001$ & 0,55 \\
\hline
\end{tabular}

Nota. $N=269$. FCCS = Scale Fear Contracting COVID-19; EADS-21 = Escalas de Ansiedade, Depressão e Stresse.

\section{Discussão}

Atendendo à pandemia pela COVID-19, avaliar o medo de contrair esta doença tornou-se um aspeto fundamental, pelo que Trindade e Ferreira (2020) desenvolveram a Fear of Contracting COVID Scale, tendo estudado a sua estrutura fatorial e propriedades psicométricas em adultos com doença inflamatória intestinal. Dado que este instrumento não havia sido ainda usado em adolescentes, o objetivo do presente estudo foi o de explorar a adequação deste instrumento para esta população. Adicionalmente, procurou- 
se analisar a relação entre o medo de contrair a COVID-19, sintomas psicopatológicos (ansiedade, depressão e stresse) e qualidade de vida nos adolescentes, bem como explorar a eventual existência de diferenças entre rapazes e raparigas no que se refere a estas variáveis.

Tal como havia sucedido na versão da FCCS para adultos (Trindade \& Ferreira, 2020), também na população adolescente se observou que este instrumento apresentava uma estrutura unidimensional, tendo este modelo revelado, globalmente, um bom ajustamento aos dados (Marôco, 2010). Ainda assim, é de referir que os Itens 2 ("Sair de casa"), 3 ("Estar com pessoas") e 6 ("Contactar com profissionais de saúde") apresentaram valores de ajustamento local, avaliados através dos pesos de regressão estandardizados e das correlações múltiplas ao quadrado, inferiores aos valores recomendados (Marôco, 2014). Com efeito, se atendermos ao conteúdo dos referidos itens considerando o período de recolha de dados, no qual havia sido iniciado o desconfinamento, com o regresso ao ensino presencial, as situações espelhadas nos itens podem ser lidas como envolvendo um menor contributo para a experiência de medo de contrair a COVID-19. Aquando da sua participação no estudo, os adolescentes estavam já a frequentar as aulas na escola e atividades extracurriculares de natureza diversa, o que envolveu a realização de testes à COVID-19 e, consequentemente, o contacto com profissionais de saúde.

A FCCS, estudada originalmente em adultos com diagnóstico de doença inflamatória intestinal, evidenciou uma boa consistência interna (Trindade \& Ferreira, 2020). Contudo, atendendo aos resultados de consistência interna encontrados no presente estudo, o uso da FCCS parece adequar-se a adolescentes no que se refere à medição do constructo do medo de contrair a infeção provocada pelo SARS-CoV-2, contribuindo para a avaliação psicológica de adolescentes em contexto de pandemia.

Os dados obtidos no presente estudo revelaram que maiores níveis de medo de contrair a COVID-19 estão relacionados com a existência de sintomas psicopatológicos de ansiedade, depressão e stresse, e que as raparigas apresentam valores mais elevados comparativamente com os rapazes. Este padrão tem sido reportado em diversos estudos (e. g., Nabuco, 2020; Ornell et al., 2020; Paulino, 2021; Wang et al., 2020). Adicionalmente, Magson et al. (2020), num estudo longitudinal em adolescentes aquando do primeiro confinamento, apontam para um aumento dos níveis de ansiedade, depressão e stresse e para uma diminuição da satisfação com a vida, particularmente nas raparigas.

De notar que uma das medidas efetuadas pelo governo português para garantir a proteção da população, desacelerar a tendência crescente de transmissão e impedir o colapso dos serviços de saúde foi o fecho dos estabelecimentos de ensino, tendo este ocorrido num primeiro confinamento iniciado em março de 2020 e num segundo confinamento, em que o encerramento das escolas se manteve entre 22 de janeiro e 05 de abril de 2021 para os $2^{\circ}$ e $3^{\circ}$ ciclos do ensino básico. De acordo com Oliveira et al. (2020), a interrupção das rotinas diárias, o método do ensino e de avaliação à distância, o confinamento em casa, os afastamentos do grupo de pares, entre outras circunstâncias, terão gerado nas crianças e adolescentes medos, incertezas, ansiedade, distanciamento social dos pares ou amigos, podendo ter afetado significativamente o seu bem-estar e a sua qualidade de vida. No entanto, no presente estudo o medo de contrair a COVID-19 mostrou-se associado a sintomas psicopatológicos de ansiedade, depressão e stresse, mas não evidenciou uma associação com a qualidade de vida. Por um lado, há que ter em conta que o medo de contrair a doença poderá não se constituir como um elemento nuclear das múltiplas consequências decorrentes da pandemia (com maior impacto na qualidade de vida). Por outro lado, hipotetizamos que as características da situação pandémica em Portugal nos meses em que ocorreu a recolha de dados do presente estudo possam ter influenciado estes resultados. Com efeito, os meses de abril e maio pautaram- 
se por medidas governamentais de desconfinamento, havendo o regresso à escola, às aulas, à rotina diária e ao encontro presencial com os pares. Estas alterações podem ter contribuído para atenuar o medo de contrair a doença e conduzido a uma perceção da qualidade de vida mais positiva. De acrescentar que, segundo dados oficiais da Direção Geral da Saúde (2020), no mesmo período, observou-se uma redução do número de casos confirmados de infeção pelo SARS-CoV-2 nos concelhos de Aveiro e de ílhavo, nos quais a recolha de dados do presente estudo foi realizada. Mais precisamente, em Aveiro, de 52 casos confirmados no mês de abril houve uma redução para 34 casos no mês de maio, e em Ílhavo, de 39 casos confirmados no mês de abril, verificou-se uma diminuição para três casos no mês maio.

\section{Limitações e Conclusão}

Os resultados do presente estudo devem ser interpretados atendendo a limitações metodológicas. Tratando-se de uma amostra por conveniência, não está garantida a representatividade da população. Para além disso, o desenho transversal do estudo não possibilita o estabelecimento de relações causais e, neste caso em concreto, pode ainda ter sofrido a influência de fatores relacionados com a própria evolução da situação pandémica e medidas sanitárias associadas. Assim, sugere-se que futuramente possam ser adotados desenhos longitudinais, passíveis de contemplar diferenças decorrentes das circunstâncias e medidas de saúde pública adotadas, assim como o recurso a uma amostra de maior dimensão, proveniente de diferentes zonas geográficas de Portugal.

Apesar das limitações apontadas, o presente estudo contribuiu metodologicamente para o estudo sobre o medo de contrair a COVID-19 em adolescentes, bem como da sua relação com a ansiedade, depressão, stresse e qualidade de vida nesta faixa etária. De referir que a disponibilidade de um instrumento de avaliação do medo de contrair a COVID-19, validado para adolescentes, possibilita a realização de estudos futuros nesta população. Esta utilidade torna-se relevante face à indefinição temporal da circunstância da atual pandemia pela COVID-19, inclusivamente com o surgimento de novas variantes do vírus, bem como das consequências deste fenómeno nos adolescentes. De acrescentar que do ponto de vista da saúde pública, este instrumento poderá funcionar como um ponto de partida para o desenvolvimento de outros similares, que possibilitem a avaliação em saúde, nomeadamente em situações de doenças transmissíveis.

\section{Conflito de interesses | Conflict of interest: nenhum | none.}

Fontes de financiamento | Funding sources: nenhuma | none.

Contributos: MFSR: Pesquisa bibliográfica; Revisão da literatura; Recrutamento dos participantes; Análise estatística; Escrita do manuscrito. AG: Desenho do estudo; Preparação das medidas e escrita do protocolo; Análise estatística; Supervisão da escrita do manuscrito e aprovação do manuscrito final. MC: Preparação das medidas e escrita do protocolo; Revisão e aprovação do manuscrito final; IMC: Análise estatística; Revisão e aprovação do manuscrito final.

\section{Referências}

Ahorsu, D. K., Lin, C. Y., Imani, V., Saffari, M., Griffiths, M. D., \& Pakpour, A. H. (2020). The fear of COVID-19 scale: Development and initial validation. International Journal of Mental Health and Addiction, 1-9. https://doi.org/ggq8b9 
Alcobia, I., Claro, C., \& Esteves, M. L. (2020). O olhar das crianças/adolescentes sobre a pandemia Covid-19 e a psicologia. Revista INFAD de Psicología. International Journal of Developmental and Educational Psychology, 2(1), 249-256. https://doi.org/g36s

Brooks, S. K., Webster, R. K., Smith, L. E., Woodland, L., Wessely, S., Greenberg, N., \& Rubin, G. J. (2020). The psychological impact of quarantine and how to reduce it: Rapid review of the evidence. The Lancet. 395(10227), 912-920. https://doi.org/ggnth8

Brito, I. (2011). Ansiedade e depressão na adolescência. Revista Portuguesa de Medicina Geral e Familiar, 27(2), 208-214. https://bit.ly/3GRGJKV

Cao, W., Fang, Z., Hou, G., Han, M., Xu, X., Dong, J., \& Zheng, J. (2020). The psychological impact of the COVID19 epidemic on college students in China. Psychiatry Research, 287, 112934. https://doi.org/ggq78v

Cohen, J. (1992). Statistical power analysis. Current Directions in Psychological Science, 1(3), 98-101. https://doi.org/cxqf2s

Correia, D. T., \& Brites J. A. (2020). Guia prático para vencer a ansiedade. Bertrand Editora.

Costello, A. B., \& Osborne, J. (2005). Best practices in exploratory factor analysis: Four recommendations for getting the most from your analysis. Practical Assessment, Research, and Evaluation, 10(1), 1-9. https://doi.org/ghgv6m

Direção Geral da Saúde. (2020). Relatório de situação. https://bit.ly/3CuAJ82

Gaspar, T., Matos, M. G. D., Ribeiro, J. L. P., \& Leal, I. (2006). Qualidade de vida e bem-estar em crianças e adolescentes. Revista Brasileira de Terapias Cognitivas, 2(2), 47-60. https://bit.ly/3GQGXSm

Gaspar, T., \& Matos, M. G. (2008). Qualidade de vida em crianças e adolescentes: Versão portuguesa dos instrumentos KIDSCREEN-52. Aventura Social e Saúde.

Gaspar, T., Ribeiro, J. L. P., Matos, M. G., \& Leal, I. (2008). Promoção de qualidade de vida em crianças e adolescentes. Psicologia, Saúde \& Doenças, 9(1), 55-71. https://bit.ly/3BRddRQ

Hamoda, H. M., Chiumento, A., Alonge, O., Hamdani, S. U., Saeed, K., Wissow, L., \& Rahman, A. (2021). Addressing the consequences of the COVID-19 lockdown for children's mental health: Investing in school mental health programs. Psychiatric Services, 72(6), 729-731. https://doi.org/g36q

Hu, L., \& Bentler, P. M. (1999). Cut-off criteria for fit indexes in covariance, structure analysis: Conventional criteria versus new alternatives. Structural Equation Modelling: A Multidisciplinary Journal, 6(1), 1-55. https://doi.org/dbt

Jiao, W. Y., Wang, L. N., Liu, J., Fang, S. F., Jiao, F. Y., Pettoello-Mantovani, M., \& Somekh, E. (2020). Behavioral and emotional disorders in children during the COVID-19 epidemic. The Journal of Pediatrics, 221, 264-266. https://doi.org/ggv5m9

Kline, R. B. (2000). Principles and practice of structural equation modelling ( $\left(2^{\mathrm{a}}\right.$ ed.). The Guilford Press.

Kline, T. J. B. (2005). Psychological testing: A practical approach to design and evaluation. Sage Publications.

Lee, S. A. (2020a). Coronavirus Anxiety Scale: A brief mental health screener for COVID-19 related anxiety. Death Studies, 44(7), 393-401. https://doi.org/ggv45c

Lee, S. A. (2020b). How much "Thinking" about COVID-19 is clinically dysfunctional? Brain, Behavior, and Immunity, 87, 97-98. https://doi.org/ggv3nc

Magalhães, A. (2007). A psicologia das emoções: O fascínio do rosto humano. Edições Universidade Fernando Pessoa.

Magson, N. R., Freeman, J. Y., Rapee, R. M., Richardson, C. E., Oar, E. L., \& Fardouly, J. (2020). Risk and protective factors for prospective changes in adolescent mental health during the COVID-19 pandemic. Journal of Youth and Adolescence, 50(1), 44-57. https://doi.org/gj277j

Marôco, J. (2010). Análise de equações estruturais: Fundamentos teóricos, software \& aplicações. ReportNumber.

Marôco, J. (2014). Análise estatística com o SPSS Statistics (7ª Ed.). Report Number, Lda.

Matos, M. G. D., Gaspar, T., \& Simões, C. (2012). Health-related quality of life in Portuguese children and adolescents. Psicologia: Reflexão e Crítica, 25(2), 230-237. https://doi.org/g34q

Masuyama, A., Shinkawa, H., \& Kubo, T. (2020). Validation and psychometric properties of the Japanese version of the fear of COVID-19 scale among adolescents. International Journal of Mental Health and Addiction, 111. https://doi.org/gg4vkc

Matos, M. G. D., Gaspar, T., \& Simões, C. (2012). Health-related quality of life in Portuguese children and adolescents. Psicologia: Reflexão e Crítica, 25(2), 230-237. https://doi.org/g34q 
Merino V. A. \& Agustin N. M. (2020). Analysis of the stress, anxiety and healthy habits in the Spanish COVID19 confinement. Health Science Journal, 14(2), 1-6. https://bit.ly/3o3Apay

Miliauskas, C. R., \& Faus, D. P. (2020). Saúde mental de adolescentes em tempos de Covid-19: desafios e possibilidades de enfrentamento. Physis: Revista de Saúde Coletiva, 30, e300402. https://doi.org/gkrrkh

Nabuco, G., de Oliveira, M. H. P. P., \& Afonso, M. P. D. (2020). O impacto da pandemia pela COVID-19 na saúde mental. Revista Brasileira de Medicina de Família e Comunidade, 15(42), 2532-2532. https://doi.org/10.5712/rbmfc15(42)2532

Oliveira, W. A. D., Silva, J. L. D., Andrade, A. L. M., Micheli, D. D., Carlos, D. M., \& Silva, M. A. I. (2020). A saúde do adolescente em tempos da COVID-19: Scoping review. Cadernos de Saúde Pública, 36, e00150020. https://doi.org/gk7k7h

Ordem dos Psicólogos Portugueses. (2014). Investir na prevenção e promoção da saúde mental em contexto educativo. http://recursos.ordemdospsicologos.pt/files/artigos/investir_na_prev_e_prom_da_sp_em_c_edu.pdf

Ornell, F., Schuch, J. B., Sordi, A. O., \& Kessler, F. H. P. (2020). Pandemia de medo e Covid-19: impacto na saúde mental e possíveis estratégias. Revista Debates in Psychiatry, 42(3), 232-235. https://doi.org/ggr2rn

Pais-Ribeiro, J. L. P., Honrado, A. A. J. D., \& Leal, I. (2004). Contribuição para o estudo da adaptação portuguesa das escalas de ansiedade, depressão e stress (EADS) de 21 itens de Lovibond e Lovibond. Psicologia, Saúde \& Doenças, 5(2), 229-239. https://bit.ly/2ZW9Ifu

Paulino, M., Dumas-Diniz, R., Brissos, S., Brites, R., Alho, L., Simões, M. R., \& Silva, C. F. (2021). COVID-19 in Portugal: exploring the immediate psychological impact on the general population. Psychology, Health \& Medicine, 26(1), 44-55. https://doi.org/f3s3

Pestana, M., \& Gageiro, J. (2020). Análise de dados para ciências sociais: A complementaridade do SPSS. Edições Sílabo.

Ravens-Sieberer, U., Gosch, A., Rajmil, L., Erhart, M., Bruil, J., Duer, W., Auquier, P., Power, M., Abel, T., Czemy, L., Mazur, J., Czimbalmos, A., Tountas, Y., Hagquist, C., Kilroe, J., \& European KIDSCREEN Group (2005). KIDSCREEN-52 quality-of-life measure for children and adolescents. Expert Review of Pharmacoeconomics \& Outcomes Research, 5(3), 353-364. https://doi.org/b5bprf

Reeve, J. (2014). Understanding motivation and emotion. John Wiley \& Sons.

Rogers, R. W. (1975). A protection motivation theory of fear appeals and attitude change. The Journal of Psychology: Interdisciplinary and Applied, 91(1), 93-114. https://doi.org/cb4jgn

Salvaterra, F., \& Chora, M. (2021). Relatório de investigação. https://bit.ly/3nB5vGo

Schmidt, B., Crepaldi, M. A., Bolze, S. D. A., Neiva-Silva, L., \& Demenech, L. M. (2020). Impactos na saúde mental e intervenções psicológicas diante da pandemia do novo coronavírus (COVID-19). Estudos de Psicologia, 37, e200063. https://doi.org/ggxdwt

Schoen, T. H., \& Vitalle, M. S. S. (2012). What am I afraid of? Revista Paulista de Pediatria, 30(1), 72-78. https://doi.org/g43j

Singh, S., Roy, M. D., Sinha, C. P. T. M. K., Parveen, C. P. T. M. S., Sharma, C. P. T. G., \& Joshi, C. P. T. G. (2020). Impact of COVID-19 and lockdown on mental health of children and adolescents: A narrative review with recommendations. Psychiatry Research, 293, 113429. https://doi.org/ghj8xn

Soares, A. H. R., Martins, A. J., Lopes, M. D. C. B., Britto, J. A. A. D., Oliveira, C. Q. D., \& Moreira, M. C. N. (2011). Qualidade de vida de crianças e adolescentes: uma revisão bibliográfica. Ciência \& Saúde Coletiva, 16, 31973206. https://doi.org/g34p

Soares, F. R., Afonso, R. M., Martins, A. P., Pakpour, A. H., \& Rosa, C. P. (2021). The fear of the COVID-19 Scale: validation in the Portuguese general population. Death Studies, 1-7. https://doi.org/10.1080/07481187.2021.1889722

Tabachnick, B. G., \& Fidell, L. S. (2013). Using multivariate statistics. Pearson Education.

Taylor, S., Landry, C. A., Paluszek, M. M., Fergus, T. A., McKay, D., \& Asmundson, G. J. (2020). Development and initial validation of the COVID Stress Scales. Journal of Anxiety Disorders, 72, 102232. https://doi.org/10.1016/j.janxdis.2020.102232

Trindade, I. A., \& Ferreira, N. B. (2020). COVID-19 Pandemic's effects on disease and psychological outcomes of people with inflammatory bowel disease in Portugal: A preliminary research. Inflammatory Bowel Diseases. 27(8), 1224-1229. https://doi.org/10.1093/ibd/izaa261 
Wallander, J. L., Schmitt, M., \& Koot, H. M. (2001). Quality of life measurement in children and adolescents: issues, instruments, and applications. Journal of Clinical Psychology, 57(4), 571-585. https://doi.org/10.1002/jclp.1029

Wang, C., Pan, R., Wan, X., Tan, Y., Xu, L., Ho, C. S., \& Ho, R. C. (2020). Immediate psychological responses and associated factors during the initial stage of the 2019 coronavirus disease (COVID-19) epidemic among the general population in China. International Journal of Environmental Research and Public Health, 17(5). https://doi.org/ggpxx6

Wise, T., Zbozinek, T. D., Michelini, G., Hagan, C. C., \& Mobbs, D. (2020). Changes in risk perception and selfreported protective behaviour during the first week of the COVID-19 pandemic in the United States. Royal Society Open Science, 7(9), 200742. https://doi.org/10.1098/rsos.200742

World Health Organization. Division of Health Promotion, Education, and Communication. (1998). Promoción de la salud: glosario. https://apps.who.int/iris/handle/10665/67246

World Health Organization. (2020) Coronavirus disease (COVID-19) pandemic. https://bit.ly/3GxdBZa 